\title{
Comparison of two-, three-, and four-exposure quadrature phase-shifting holography
}

\author{
Jung-Ping Liu, ${ }^{1, *}$ Ting-Chung Poon, ${ }^{1,2}$ Gui-Syu Jhou, ${ }^{1}$ and Po-Jung Chen ${ }^{1}$ \\ 'Department of Photonics, Feng Chia University, No. 100 Wenhwa Road, Taichung 407, Taiwan \\ ${ }^{2}$ Bradley Department of Electrical and Computer Engineering, Virginia Tech, Blacksburg, Virginia 24061, USA \\ ${ }^{*}$ Corresponding author: jpliu@fcu.edu.tw
}

Received 18 November 2010; revised 26 February 2011; accepted 16 March 2011; posted 18 March 2011 (Doc. ID 138365); published 27 May 2011

\begin{abstract}
In standard (four-exposure) quadrature phase-shifting holography (QPSH), two holograms and two intensity maps are acquired for zero-order-free and twin-image-free reconstruction. The measurement of the intensity map of the object light can be omitted in three-exposure QPSH. Furthermore, the measurements of the two intensity maps can be omitted in two-exposure QPSH, and the acquisition time of the overall holographic recording process is reduced. In this paper we examine the quality of the reconstructed images in two-, three-, and four-exposure QPSH, in simulations as well as in optical experiments. Various intensity ratios of the object light and the reference light are taken into account. Simulations show that two- and three-exposure QPSH can provide reconstructed images with quality comparable to that of four-exposure QPSH at a low intensity ratio. In practice the intensity ratio is limited by visibility, and thus four-exposure QPSH exhibits the best quality of the reconstructed image. The uniformity and the phase error of the reference light are also discussed. We found in most cases there is no significant difference between the reconstructed images in two- and three-exposure QPSH, and the quality of the reconstructed images is acceptable for visual applications such as the acquisition of three-dimensional scene for display or particle tracking. () 2011 Optical Society of America
\end{abstract}

OCIS codes: $\quad 090.0090,090.1995$.

\section{Introduction}

In the past decade, digital holography was widely investigated for its potential applications of metrology, three-dimensional display and holographic microscopy $[1,2]$. In digital holography, the interferogram is acquired and recorded electronically. Since there is always the zero-order term and the twin image in a single interferogram, one has to remove them before further processing. There are some methods to remove the zero-order term and the twin image from a single interferogram, such as using off-axis configuration $[3,4]$ or using Hilbert digital phase shifting [5]. Nevertheless, they are limited to specific configurations or specimens. Typically, multiple interferograms are required for removing the zero-order term and the twin image, and phase-shifting holography

0003-6935/11/162443-08\$15.00/0

(C) 2011 Optical Society of America
(PSH) [6,7] is one of the most common techniques. In conventional PSH, three or more phase-shifted interferograms are used for zero-order-free and twin-image-free reconstruction [8]. In 2004, Guo and Devaney [9] demonstrated four-exposure quadrature phase-shifting holography (QPSH) in which only two interferograms with $\pi / 2$ phase shift plus two intensity measurements are needed. The fourexposure QPSH was then generalized by Meng et al. in that the phase shift can be arbitrary between 0 and $\pi[10]$. However, the accurate amount of phase shift should be known prior the digital reconstruction, or the error of the supposed phase shift will result in additional noise in the reconstructed image. To measure and calibrate the phase error, Chen et al. proposed three different iterative algorithms [11], and $\mathrm{Xu}$ et al. proposed a noniterative algorithm so that the computation time can be reduced significantly [12]. Also based on the four-exposure QPSH, 
Meng et al. [13] proposed three-exposure QPSH in which the intensity distribution of the object light is calculated so that the number of exposures is reduced to three. If the zero-order light is uniform across the charge-coupled device (CCD) plane, the low-pass filtered interferograms can be regarded as the zeroorder term, and hence the number of exposures can be reduced to two [14]. On the other hand, Shaked et al. suggested suppressing the zero-order term by QPSH, but to separate the twin-image term by an off-axis configuration. Therefore, only two exposures are required, and no complicated algorithm is required in the technique [15].

Recently Liu and Poon proposed two-exposure QPSH in which an estimation algorithm is applied to find the intensity value of the reference light [16]. Combining the algorithm of three-exposure QP $\overline{\mathrm{SH}}$, only two exposures are required to remove the zeroorder term and the twin image. There are, however, some limitations in three-exposure and two-exposure QPSH. First, the reference light must be normal incident, and its intensity must be larger than the intensity of the object light. Moreover, in two-exposure QPSH, the reference light is assumed to be uniform over the whole hologram, which is not so easy fulfill in practice. In this paper we will compare the quality of the reconstructed images in two-, three-, and fourexposure QPSH, in various practical conditions. In Section 2, the principle of QPSH is briefly reviewed. In Section 3, we compare four types of QPSH in simulations. The intensity ratio, the uniformity of the reference light, and the error of the phase shift are taken into account. We will provide experimental demonstrations in Section $\underline{4}$. Finally, in Section $\underline{5}$ we make some concluding remarks.

\section{Principle of QPSH}

Figure 1 depicts a typical setup of QPSH. A collimated laser beam is separated into two, the object arm and the reference arm. In the object arm, the object target is illuminated with the collimated laser beam, and the object light is combined with the reference light by a beam splitter (BS). The interference pattern on the chip of the CCD is acquired as an interferogram. In the reference arm, a plate of glass is inserted to play the role of phase shifter. Two interferograms of $\pi / 2$ phase shift with each other can be produced by slightly rotating the plate. The two interferograms can be expressed as

$$
I_{H i}=I_{O}+I_{R}+O \cdot R^{*} e^{-j \theta_{i}}+O^{*} \cdot R e^{j \theta_{i}}, \quad \text { for } i=1,2,
$$

where $O=|O| e^{j \phi_{o}}$ is the complex amplitude of the object light, $R=|R| e^{j \phi_{r}}$ is the complex amplitude of the reference light, $I_{O}=|O|^{2}$ and $I_{R}=|R|^{2}, \phi_{o}$ and $\phi_{r}$ are the phase of the object light and the reference light, respectively, and $\theta_{i}$ is the phase induced by the phase shifter so that $\theta_{1}=0$ and $\theta_{2}=\pi / 2$ for QPSH. From Eq. (1) and after some manipulations, one can obtain a quadratic equation:

$$
\begin{aligned}
& 2\left(I_{O}+I_{R}\right)^{2}-\left(4 I_{R}+2 I_{H 1}+2 I_{H 2}\right)\left(I_{O}+I_{R}\right) \\
& \quad+\left(I_{H 1}^{2}+I_{H 2}^{2}+4 I_{R}^{2}\right) \\
& \quad=0
\end{aligned}
$$

and the zero-order term, $\left(I_{O}+I_{R}\right)$, can be easily found by

$$
\begin{aligned}
I_{O}+I_{R}= & \frac{2 I_{R}+I_{H 1}+I_{H 2}}{2} \\
& -\frac{\sqrt{\left(2 I_{R}+I_{H 1}+I_{H 2}\right)^{2}-2\left(I_{H 1}^{2}+I_{H 2}^{2}+4 I_{R}^{2}\right)}}{2} .
\end{aligned}
$$

From Eq. (3), we see that the intensity of the object light can be found from $I_{R}, I_{H 1}$, and $I_{H 2}$. As the zero-order term $\left(I_{O}+I_{R}\right)$ is found from Eq. (3), the complex hologram without the zero order and the twin image can be calculated digitally by

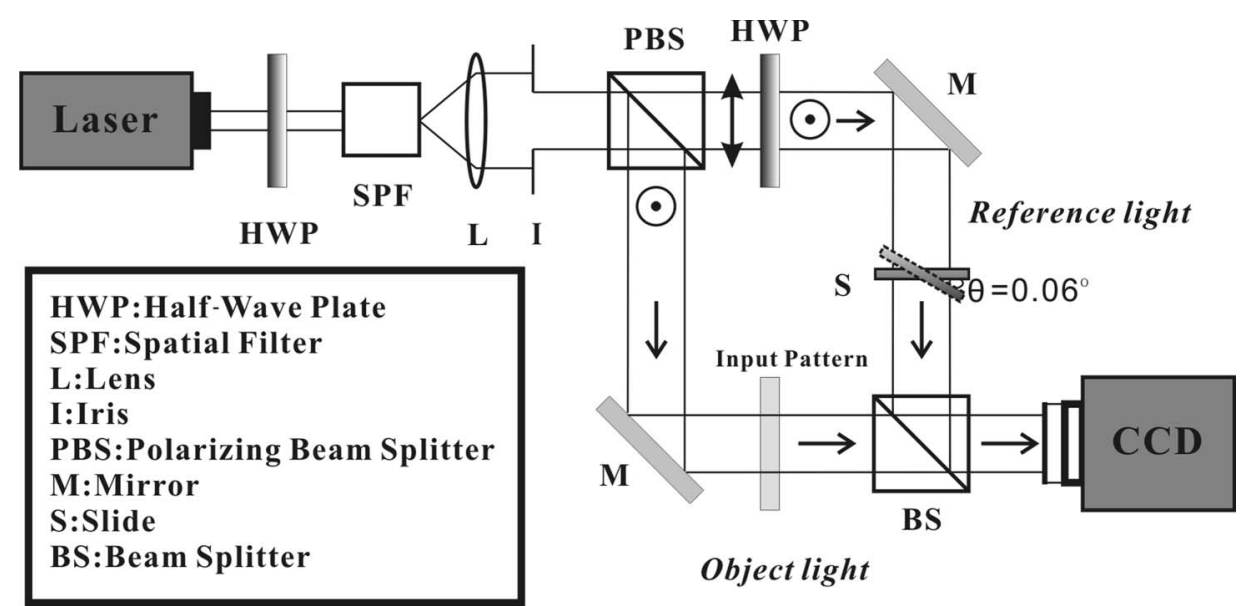

Fig. 1. Schematic diagram of QPSH. 


$$
H_{\mathrm{QPSH}}=\frac{\left(I_{H 1}-I_{O}-I_{R}\right)+j\left(I_{H 2}-I_{O}-I_{R}\right)}{2}=O R^{*} .
$$

In the present formulas the reference light is not limited to normal incident or uniformly distributed. Therefore, the term $R^{*}$ in the right side of Eq. (4) cannot be regarded as a constant unless the reference light is a normal-incident plane wave. For any off-axis reference plane wave incidence, an additional process such as removing the carrier frequency is needed.

In four-exposure QPSH, $I_{H 1}, I_{H 2}, I_{R}$, and $I_{O}$ are measured sequentially, and the complex hologram is calculated according to Eq. (4). In three-exposure QPSH, the zero-order term is calculated according to Eq. (3), and hence the measurement of $I_{O}$ is not required. In two-exposure QPSH, the intensity of the reference light is assumed to be uniform so that it is constant across the whole CCD plane. This intensity value can be found by trial and error with the help of the normalized correlation peak (NCP),

$$
\mathrm{NCP} \equiv \frac{\operatorname{real}\left\{\left[H_{t} \otimes H_{r}\right]_{\mathrm{MAX}}\right\}}{\left[H_{r} \otimes H_{r}\right]_{\mathrm{MAX}}},
$$

where " $\otimes$ " stands for the operator of correlation, $H_{r}$ is the complex hologram obtained from Eq. (4) together with Eq. (3) at different trial intensity values $I_{r}$ of the reference light $I_{R}=|R|^{2}$ (i.e., $I_{R}=|R|^{2}$ is allowed to change), and $H_{t}$ is a target hologram that contains the information of the object field $O$. The idea in Eq. (5) is that, if $I_{r}$ is chosen correctly, $I_{r}$ becomes $I_{R}$, and NCP will achieve its maximum value. Equation (5) is also applicable for the cases involving tilted reference light if the phase of the reference light is not removed from the target hologram. In [11], the hologram $I_{H 1}$ in Eq. (1) is used as the target hologram as it contains the term $O R^{*}$. However, the zero-order and the twin-image terms are also contained in the target hologram of $I_{H 1}$, and they will affect the result of the correlation value in the NCP. In this paper, we propose an alternative target hologram in which the zero-order term is removed. The new target hologram $H_{t \_n}$ can be found from $I_{H 1}$ and $I_{H 2}$ by

$$
H_{t / n}=\frac{(1-j)}{2}\left(I_{H 1}-I_{H 2}\right)=O R^{*}-j O^{*} R .
$$

In Section $\underline{3}$, the reconstructions with $H_{t}$ and those with $H_{t \_n}$ will be examined.

\section{Simulation and Comparison}

In this section a series of simulations are conducted for the comparison of two-, three-, and four-exposure QPSH. In the simulations the wavelength is $632.8 \mathrm{~nm}$, and the hologram size is $1024 \times 1024$ pixels with pixel size of $7.4 \mu \mathrm{m}$. The object pattern is a United States Air Force resolution chart with brightness 0 and 1 , and the reference light is a normalincident plane wave. The distance between the object plane and the CCD plane is $13.3 \mathrm{~cm}$. For simplifying the description, we use A and B to stand for the fourand three-exposure QPHS, respectively; we also use $\mathrm{C}$ and $\mathrm{D}$ to stand for two-exposure QPHS with the target holograms $H_{t}$ and $H_{t \_n}$, respectively. The quality of the reconstructed image is measured by the root-mean-square error (RMSE), which is defined as

$$
\mathrm{RMSE} \equiv\left\{\frac{1}{M N} \sum_{m=1}^{M} \sum_{n=1}^{N}[F(m, n)-f(m, n)]^{2}\right\}^{1 / 2},
$$

where $F(m, n)$ stands for the ideal pattern; $f(m, n)$ stands for the estimated pattern, i.e., the intensity of the reconstructed object light at the object plane; $(m, n)$ is the pixel index, while $M$ and $N$ are the pixel number along the $x$ and $y$ axes, respectively. RMSE can be regarded as the average noise level of the estimated pattern. Thus an image with lower RMSE has better quality.

It was mentioned in [16] that Eq. (3) is valid only when $|R|$ is large enough. When Eq. (3) is not valid for all pixels of the hologram, the calculated intensity of the object light suffers from noise. This effect will be found in three- and two-exposure QPSH, but should not be found in four-exposure QPSH. The RMSE of the reconstructed images at various intensity ratios is plotted in Fig. 2. The intensity ratio is defined as

$$
\text { intensity ratio } \equiv \frac{\frac{1}{M N} \sum_{m=1}^{M} \sum_{n=1}^{N} I_{O}(m, n)}{\frac{1}{M N} \sum_{m=1}^{M} \sum_{n=1}^{N} I_{R}(m, n)}=\frac{\bar{I}_{O}}{\bar{I}_{R}},
$$

where $\bar{I}_{O}$ and $\bar{I}_{R}$ stand for the mean intensities of the object light and the reference light, respectively. Note that $I_{R}$ equals $\bar{I}_{R}$ when the reference wave is an ideal plane wave. In Fig. $\underline{2}$, curve $\mathrm{A}$ is flat at zero RMSE because the corresponding reconstruction procedure is independent of the intensity ratio. In the region of small intensity ratio, the RMSE of $B$ is as small as that of A. However, the RMSE of B increases with intensity ratio. The increasing error results from the pixels where $I_{R}$ is not large enough. In Fig. 3 , we plot the percentage of error pixels over all pixels

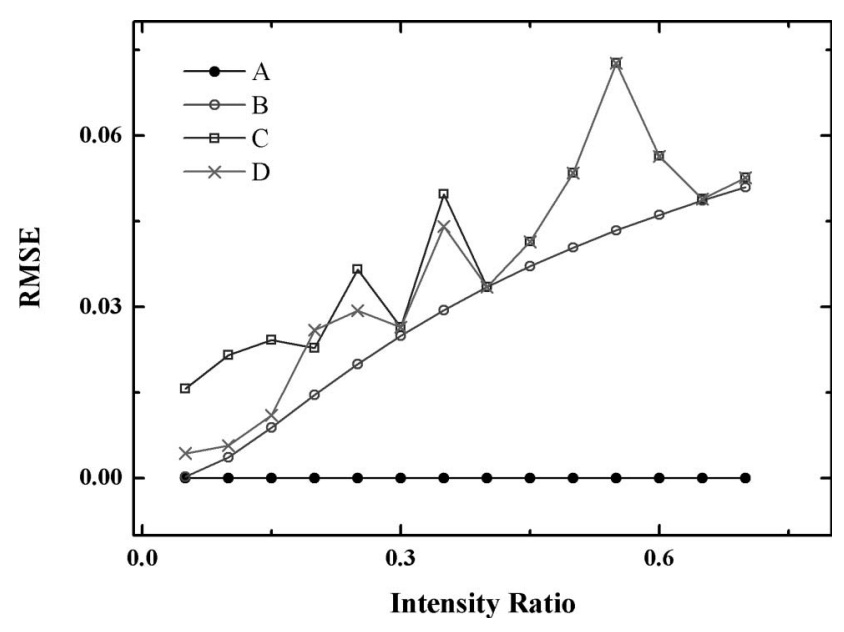

Fig. 2. RMSE as a function of intensity ratio. 


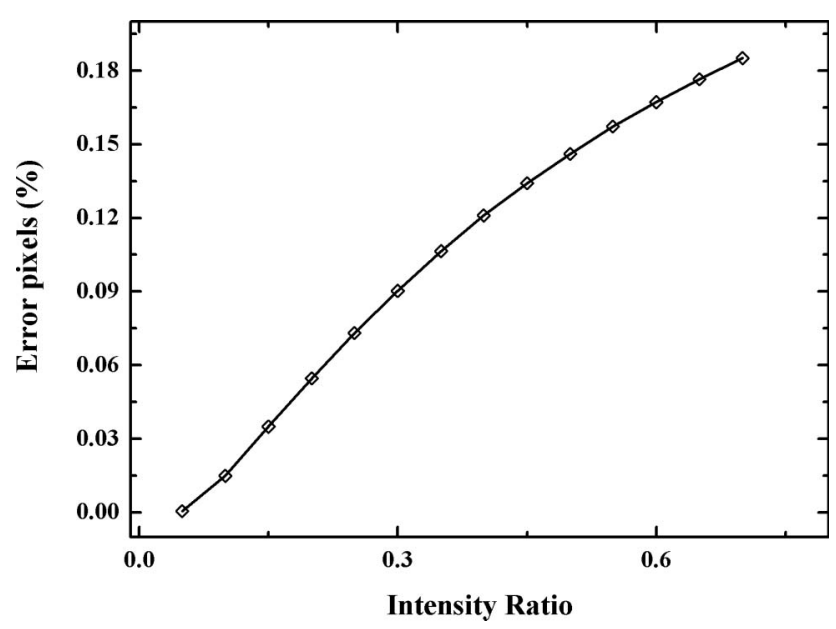

Fig. 3. Error pixels as a function of the intensity ratio for the simulation of curve B in Fig. 2.

versus the intensity ratio. Here the pixels at which the intensity of the reference light is lower than that of the object light are regarded as the error pixels. It is shown in Fig. 3 that, in the three-exposure QPSH, the error pixels increase when the intensity ratio rises. It is also shown that the RMSE is proportional to the number of error pixels. In Fig. 2 , Curve $\mathrm{C}$ is close to Curve $\mathrm{D}$, but in the region of low intensity ratio, $\mathrm{D}$ is almost smaller than $\mathrm{C}$. Thus method $\mathrm{D}$ (using $H_{t \_n}$ as the target hologram) is better than method $\mathrm{C}$ (using $I_{H 1}$ as the target hologram). Nevertheless, both $\mathrm{C}$ and $\mathrm{D}$ are equal to or worse than $\mathrm{B}$ because in method $B$ the correct intensity value of the reference light is always applied.

The error of the intensity value of the reference light found by the NCP is defined as

$$
E_{r} \equiv\left(I_{r}-\bar{I}_{R}\right) / \bar{I}_{R}
$$

and the errors corresponding to methods $\mathrm{C}$ and $\mathrm{D}$ are plotted in Fig. 4. We notice that, at some locations (e.g., $\bar{I}_{O} / \bar{I}_{R}=0 . \overline{2}$ ), $\left|E_{r}\right|$ of $\mathrm{C}$ is smaller than $\left|E_{r}\right|$ of

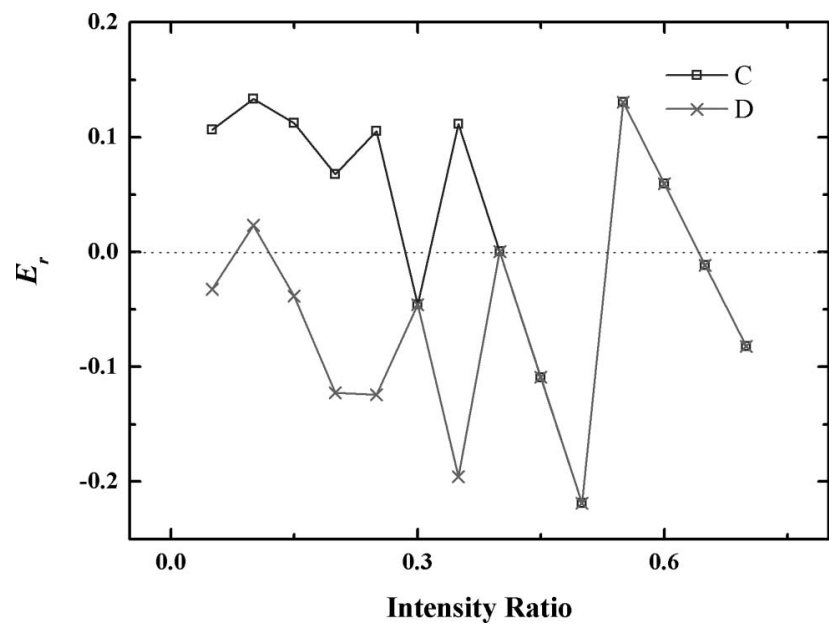

Fig. 4. Error of the calculated intensity of reference light $\left(E_{r}\right)$ as a function of the intensity ratio.

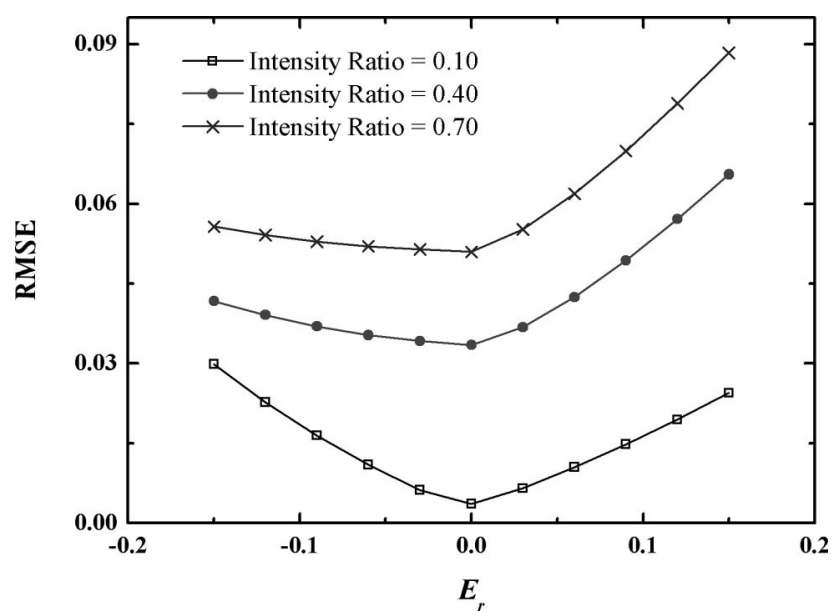

Fig. 5. RMSE as a function of $E_{r}$.

$\mathrm{D}$, but the RMSE of $\mathrm{C}$ is larger than that of $\mathrm{D}$. In other words, RMSE is not symmetrical to $E_{r}=0$. To see this effect, we plot the relation between RMSE and $E_{r}$ in Fig. $\underline{5}$. When the intensity ratio is small (0.10), RMSE is nearly symmetrical to $E_{r}=0$. When the intensity ratio is large (0.70), the tolerance in the negative region of $E_{r}$ is larger than that in the positive region. In addition, the whole RMSE curve rises as the intensity ratio becomes higher. The grown error results from the pixels at which the intensity of the reference light is not high enough. Thus for higher intensity ratio, there is always noise even though the intensity value of the reference light is correct $\left(E_{r}=0\right.$, i.e., curve B in Fig. 2).

In the above discussion, the reference light is assumed to be uniform in intensity. The assumption is hard to fulfill in practice, so the uniformity of the reference light should be discussed. Here two cases of intensity variation are discussed. The first one [Fig. 6(a)] is a Gaussian-distributed variation, while the other one [Fig. 6(b)] is a linear variation. The reference light in both cases has the same standard deviation of 0.1 over an average intensity of 1 . The simulation results corresponding to Figs. 6(a) and $6(\mathrm{~b})$ are shown in Figs. 7 and 8 , respectively. In Fig. 7 , the RMSE of methods $\mathrm{C}$ and $\overline{\mathrm{D}}$ significantly increase at the range of small intensity ratio, as compared with Fig. 2. However, in Fig. $\underline{8}$, the RMSE of methods $\mathrm{C}$ and $\mathrm{D}$ only slightly increases. The reason

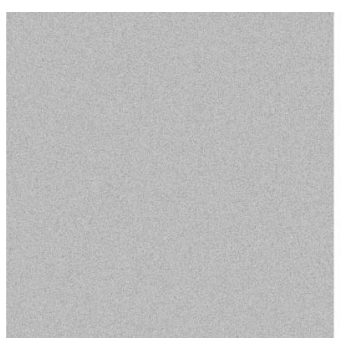

(a)

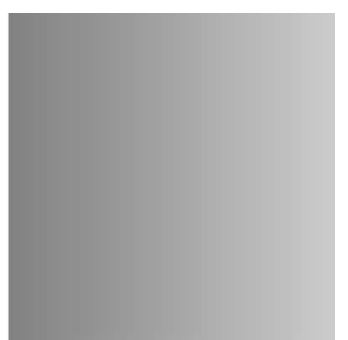

(b)
Fig. 6. Intensity distribution of the reference light with (a) Gaussian-distributed variation, (b) linear variation. 


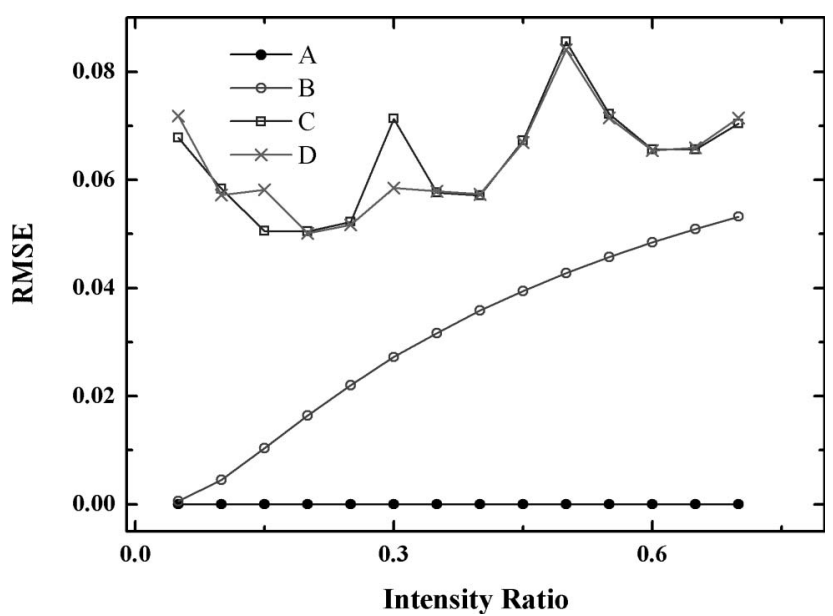

Fig. 7. RMSE as a function of intensity ratio while the reference light is the one shown in Fig. $\underline{6(a)}$.

may be explained as follows. The object light is localized in the central area so that the interference fringes are formed mainly in the central area. In addition, the intensity value in Fig. 6(a) changes severely pixel by pixel, while the change of intensity is smooth within a local area in Fig. 6(b). As a result, the effective intensity variation of the reference light in the first case is much larger than that in the second case. And extremely weak reference light is produced at some pixels in the first case, which also results in serious noise.

In QPSH, the phase difference between the reference beams for the two interferograms are supposed to be exactly $\pi / 2(1.57 \mathrm{rad})$. But in experimental implementation the phase difference may slightly deviate from $\pi / 2$. The error of the phase difference in taking the interferograms induces additional noise in the reconstructed image. Here we also conduct simulations to evaluate the effect of phase error. In the simulations two interferograms are taken using two reference lights with various phase differences, and the reconstruction is performed using the supposed phase difference, that is, $\pi / 2$. Figure $\underline{9}$ shows the

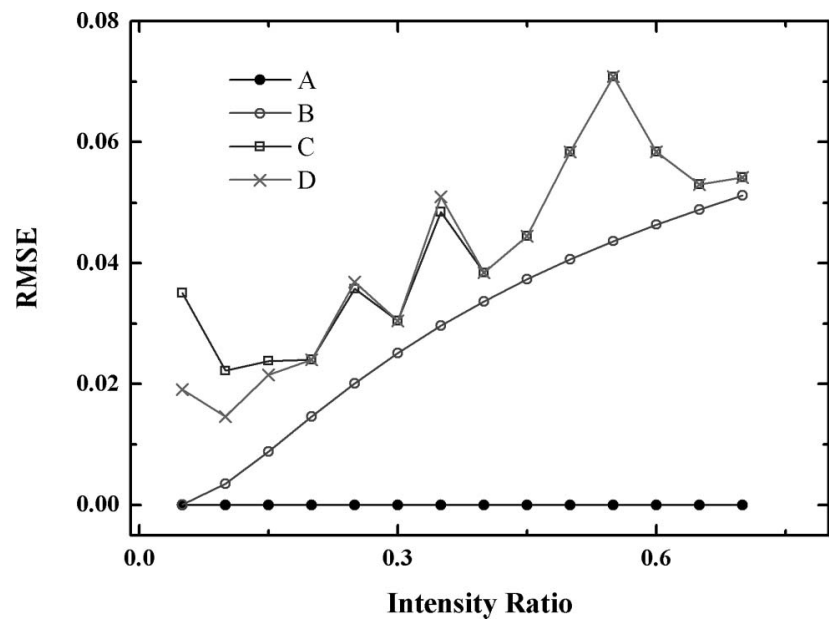

Fig. 8. RMSE as a function of intensity ratio while the reference light is the one shown in Fig. $\underline{6(\mathrm{~b})}$.

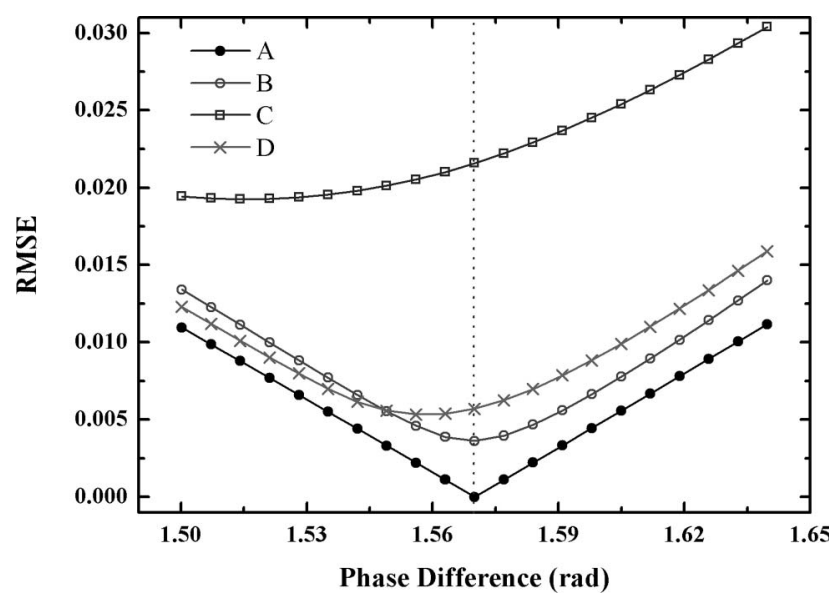

Fig. 9. RMSE as a function of phase difference while the intensity ratio is 0.1 .

RMSE as a function of the phase difference. In methods $\mathrm{A}$ and $\mathrm{B}$, the RMSE of the reconstructed image rapidly rises as the phase difference deviates from $\pi / 2$. This is because Eq. (4) no longer correct when the phase difference is not exactly $\pi / 2$. In methods $\mathrm{C}$ and $\mathrm{D}$, the minimum RMSE is not achieved at $\pi / 2$, but at the phase smaller than $\pi / 2$. In other words, we can achieve a better reconstruction using an incorrect phase difference. We notice that the error of the intensity value of the reference light evaluated by the NCP is unchanged at various phase differences, as shown in Fig. 10. Thus, it is reasoned that the phase error induces ignorable error to the zero-order term found by Eq. (3). This postulate can be verified from curves $A$ and $\bar{B}$ in Fig. 9 because the difference between them does not vary significantly at different phase errors. Moreover, the intensity value evaluated by the NCP also resists the error of phase difference because both the target hologram and the calculated complex hologram contain the same phase error. Since the estimated intensity value is independent of the phase error, the error induced by the incorrect intensity value of the

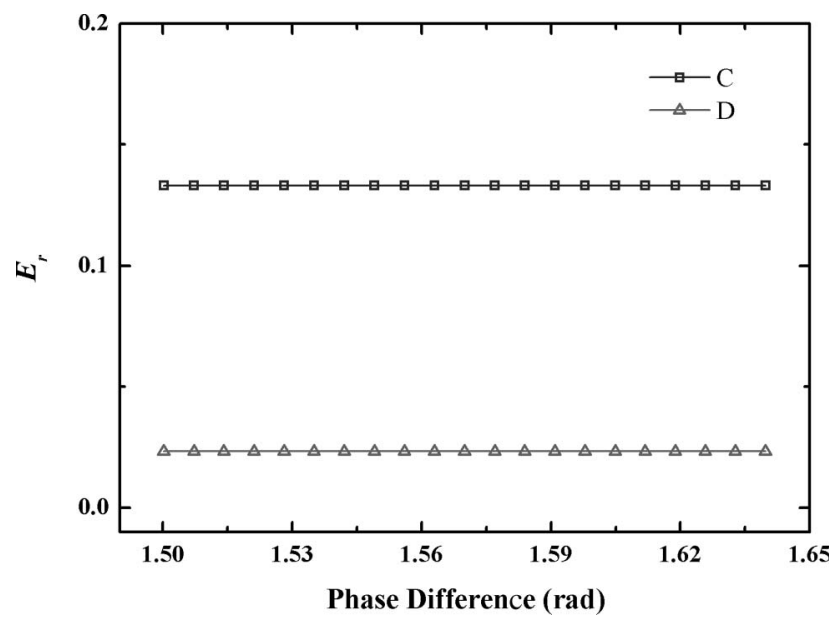

Fig. 10. $E_{r}$ as a function of phase difference while the intensity ratio is 0.1 . 
reference light can be somewhat compensated by also inducing a specified error of phase difference. Therefore a reduced RMSE can be achieved, and the larger the error of the evaluated intensity value (method C), the larger the phase error needed to achieve the minimum RMSE. Also because of the compensation, the RMSE of method D is better than that of method $\mathrm{B}$ when the phase difference is smaller than $\pi / 2$. The way of compensation is, however, hard to control because the phase error is always unknown in practice.

Finally, we also use different patterns as the objects in the simulations. The trends of the simulation results of two- and three-exposure QPSH are similar to those proposed in the paper. When the average intensity of the object light at the CCD plane varies, the RMSE curve will shift relative to the intensity ratio. Besides, the RMSE curve will rise when the contrast of the object light increase, because at a fixed intensity ratio, high-contrast object light will result in more error pixels in the reconstructed image.

\section{Experimental Demonstration}

The experimental setup is the same as shown in Fig. 1. The light source is an He-Ne laser operated at wavelength of $632.8 \mathrm{~nm}$. The laser beam is expanded and separated by a PBS. Thus, the intensity ratio of the object light and the reference light can be controlled by the combination of the half-wave plate HWP1 and PBS. In our setup, the phase shifter is a plate of glass (a slide) mounted on a rotation stage. The phase shifter produces phase retardation of $\pi / 2$ as the slide is rotated by $0.06^{\circ}$ for the thickness of the slide used in the experiment. As the two interferograms are recorded, we block the reference light and the object light by shutters (not shown in Fig. 1) to measure $I_{O}$ and $I_{R}$. The interferograms and the intensity maps are acquired by a CCD made by Apogee (model Alta U2000) with size $1600 \times 1200$ pixels and pixel pitch $7.4 \mu \mathrm{m}$. All the acquired images are cropped to a size of $1024 \times 1024$ pixels for further processing.

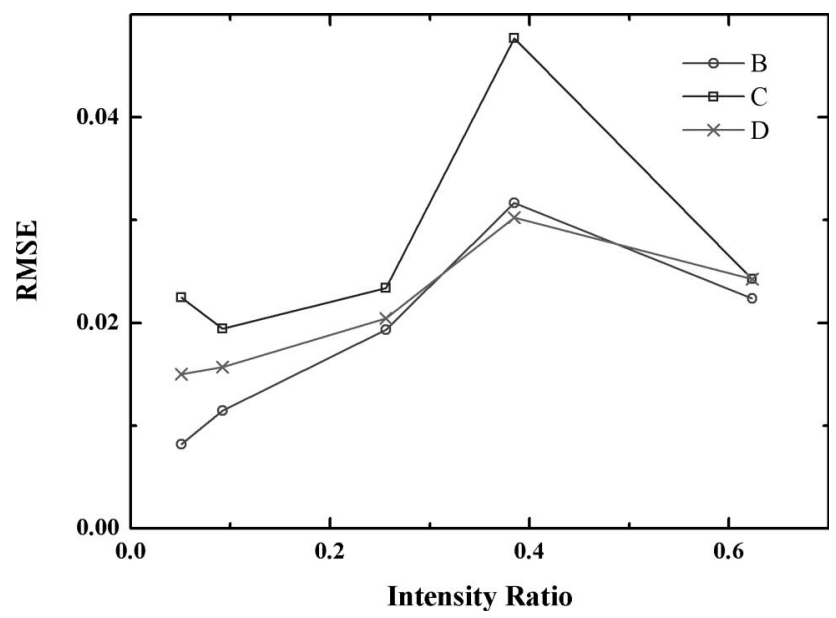

Fig. 11. RMSE as a function of intensity ratio (experiment).

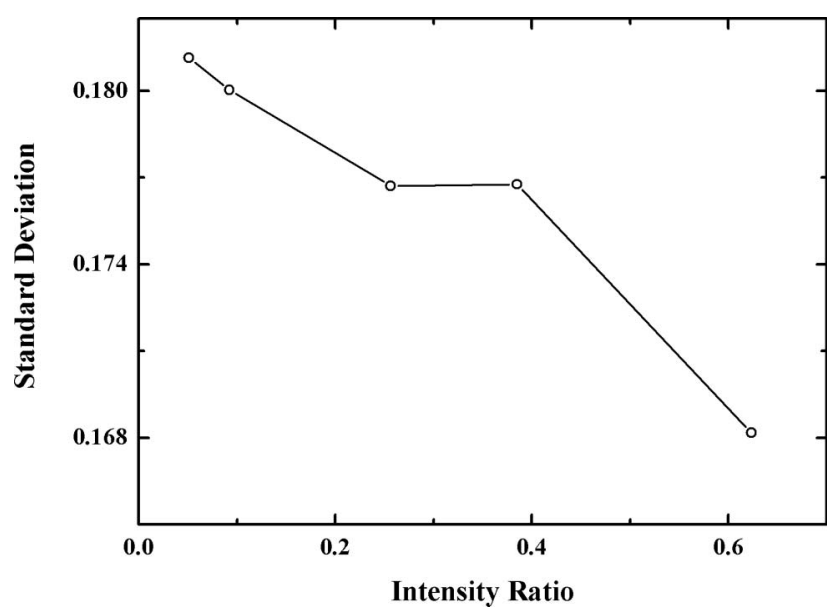

Fig. 12. Standard deviation of the measured reference light at various intensity ratios. The average brightness of all the calculated photos is normalized to unity.

Experiments at intensity ratios $(0.05,0.09,0.26$, $0.38,0.62$ ) are conducted. The RMSE of the reconstructed images at various intensity ratios is plotted in Fig. 11. Since in the experiment there is not a perfect image as the target pattern, we use the reconstructed image obtained by four-exposure QPSH as the target pattern in Eq. (7). Thus, there are only three curves in Fig. 11, and the RMSE should be read as the correlation between reconstructions of method A and method B (or C, D), not the absolute noise level. Because the reference light suffers from speckle noise and unwanted interference patterns, the standard variation of the reference light varies at different intensity ratios, as shown in Fig. 12. Thus, the curves in Fig. 11 and those in Figs. 7 or 8 cannot be compared directly. The visibility is too low to form clear interference fringes if the intensity ratio is over 0.62 or below 0.05 . We take two interferograms and

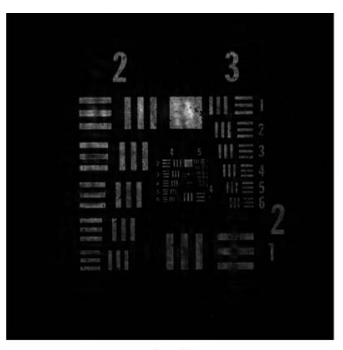

(a)

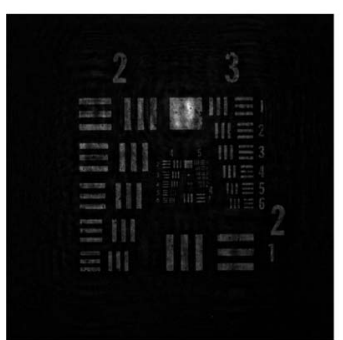

(c)

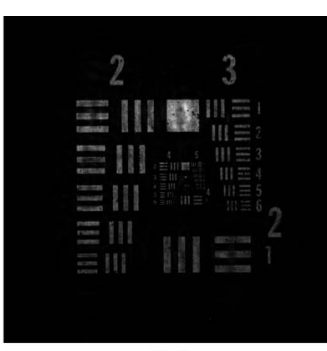

(b)

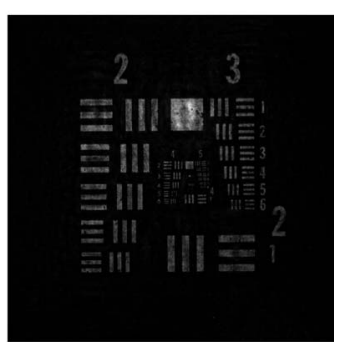

(d)
Fig. 13. Reconstructed images by methods (a) A, (b) B, (c) C, and (d) $\mathrm{D}$ while the intensity ratio is 0.05 . 


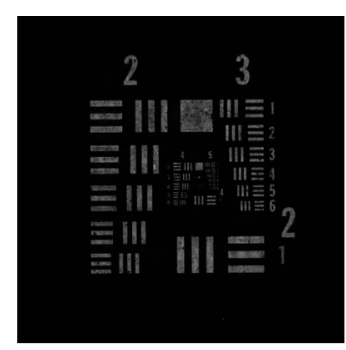

(a)

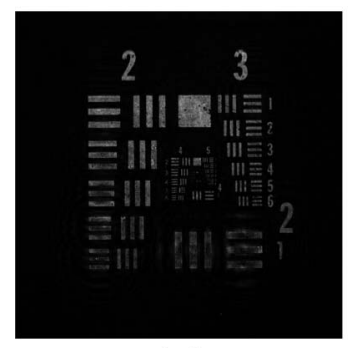

(c)

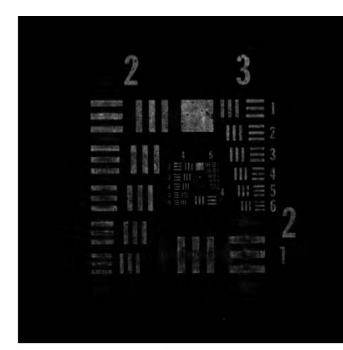

(b)

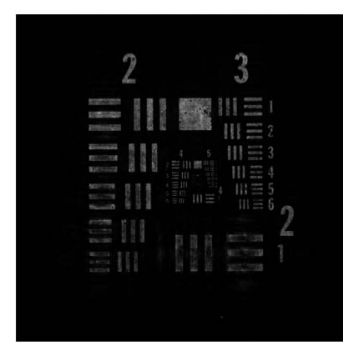

(d)
Fig. 14. Same as Fig. 13 except that the intensity ratio is 0.26 .

two intensity maps at every intensity ratio so that reconstructions by four-, three-, and two-exposure QPSH can be performed. The selected reconstructed images are shown in Figs. 13-15. At the smallest intensity ratio, namely 0.05 (Fig. $\overline{13}$ ), the reconstructed images of methods A and B are virtually the same. Reconstructions of method $\mathrm{C}$ and $\mathrm{D}$ provide similar results, and the artifacts in Fig. 13(d) seem less than those in Fig. 13(c). At a modest intensity ratio, namely 0.26 , the reconstructed image of method A [Fig. 14(a)] is the best, and the reconstructed images of methods B-D [Figs. 14(b)-14(d)] are similar to each other. Finally, at the largest intensity ratio, namely 0.62 (Fig. 15), there are significant artifacts in the reconstructed images of methods B-D. These

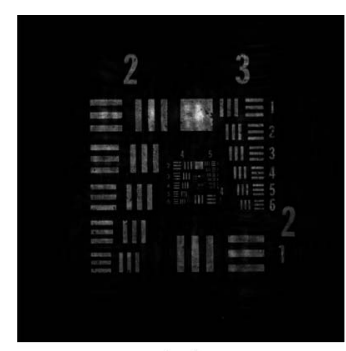

(a)

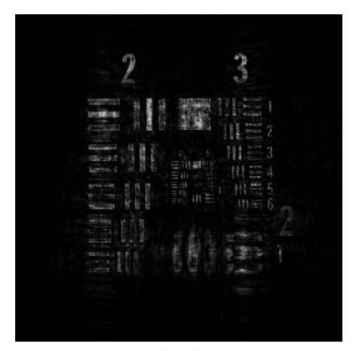

(c)

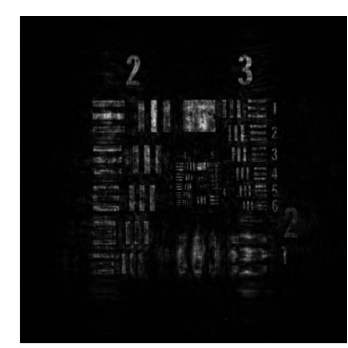

(b)

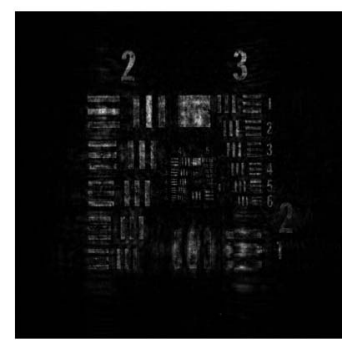

(d)
Fig. 15. Same as Fig. 13 except that the intensity ratio is 0.62 artifacts should result from the pixels where the intensity of the object light is larger than that of the reference light.

According to these experimental data, the allowable range of intensity ratio of methods $\mathrm{B}, \mathrm{C}$, and $\mathrm{D}$ is the same. The allowable range of intensity ratio of method A is only limited by visibility and thus is larger than that of methods B-D. In general cases, method D is better than method C, and method D is comparable to method $\mathrm{B}$. However, method $\mathrm{A}$ is always the best way to perform reconstruction, though it takes the most time in acquiring the holograms and intensity maps. Unlike the trend in simulations as shown in Fig. 2 , the best quality of reconstructed images in two-exposure QPSH is not achieved at the smallest intensity (0.05). It is partially due to the low visibility at a low intensity ratio. The other reason is that the standard variation of the reference light at the lowest intensity ratio is the maximum, as shown in Fig. 12 .

\section{Conclusion}

In the paper we compare two-, three-, and fourexposure QPSH in reconstruction quality. The fourexposure QPSH will take the most time in data acquisition. Nevertheless, the reconstruction quality only depends on the interference visibility. The reconstruction quality of two- and three-exposure QPSH depends not only on the visibility but also on the intensity ratio. We also found that the intensity value of the reference light calculated by twoexposure QPSH is not exactly the correct value, and thus, there is always noise produced in twoexposure QPSH. The deviation of the estimated intensity value from the correct value of the reference light results from the zero-order and the twin-image terms in the target hologram. Thus, based on the concept of hologram subtraction [17], we propose a new target hologram [Eq. (6)] in which the zero-order term is removed to reduce the estimation error. The new target hologram always exhibits a better performance than the old target hologram. The phase error in performing QPSH is also taken into account. Basically, the reconstruction quality is inversely proportional to the phase error. But for two-exposure QPSH, the reconstruction quality can be slightly improved by involving a phase error. In addition, the difference between two- and three-exposure QPSH is minor except for the case of reference light with severe Gaussian noise. Both two- and three-exposure QPSH suffer from severe noise when the intensity ratio is too high.

In the experimental demonstration, there is no significant difference between the reconstructed images in two- and three-exposure QPSH. Although fourexposure QPSH exhibits the best quality of reconstructed image, the quality of the reconstructed images in two-exposure QPSH is comparable to the three-exposure QPSH and is acceptable at a modest intensity ratio. Therefore, we suggest using fourexposure QPSH for quantitatively applications, such as contour measurement. Two-exposure QPSH can 
be well used for visual applications such as the acquisition of a three-dimensional scene for display or particle tracking.

This work is partially supported by the National Science Council of Taiwan (NSCT) under contract 99-2221-E-035-065. G.-S. Jhou and P.-J. Chen thank NSCT for awarding research scholarships to them.

\section{References}

1. T.-C. Poon, ed., Digital Holography and Three-Dimensional Display (Springer, 2006).

2. U. Schnars and W. Jueptner, Digital Holography (Springer, 2005).

3. E. Cuche, P. Marquet, and C. Depeursinge, "Simultaneous amplitude-contrast and quantitative phase-contrast microscopy by numerical reconstruction of Fresnel off-axis holograms," Appl. Opt. 38, 6994-7001 (1999).

4. E. Cuche, P. Marquet, and C. Depeursinge, "Spatial filtering for zero-order and twin-image elimination in digital off-axis holography," Appl. Opt. 39, 4070-4075 (2000).

5. W. Pan, W. Lu, Y. Zhu, and J. Wang, "One-shot in-line digital holography based Hilbert phase-shifting," Chin. Opt. Lett. 7, 1123-1125 (2009).

6. I. Yamaguchi and T. Zhang, "Phase-shifting digital holography," Opt. Lett. 22, 1268-1270 (1997).

7. T. Zhang and I. Yamaguchi, "Three-dimensional microscopy with phase-shifting digital holography," Opt. Lett. 23, 1221-1223 (1998).

8. L. Z. Cai, Q. Liu, and X. L. Yang, "Phase-shift extraction and wave-front reconstruction in phase-shifting interferometry with arbitrary phase steps," Opt. Lett. 28, 1808-1810 (2003).
9. P. Guo and A. J. Devaney, "Digital microscopy using phaseshifting digital holography with two reference waves," Opt. Lett. 29, 857-859 (2004).

10. X. F. Meng, L. Z. Cai, Y. R. Wang, X. L. Yang, X. F. Xu, G. Y. Dong, X. X. Shen, and X. C. Cheng, "Wavefront reconstruction by two-step generalized phase-shifting interferometry," Opt. Commun. 281, 5701-5705 (2008).

11. W. Chen, C. Quan, C. J. Tay, and Y. Fu, "Quantitative detection and compensation of phase-shifting error in two-step phase-shifting digital holography," Opt. Commun. 282, 2800-2805 (2009).

12. X. F. Xu, L. Z. Cai, Y. R. Wang, and R. S. Yan, "Direct phase shift extraction and wavefront reconstruction in two-step generalized phase-shifting interferometry" J. Opt. 12 , 015301 (2010).

13. X. F. Meng, L. Z. Cai, X. F. Xu, X. L. Yang, X. X. Shen, G. Y. Dong, and Y. R. Wang, "Two-step phase-shifting interferometry and its application in image encryption," Opt. Lett. 31, 1414-1416 (2006).

14. X. F. Meng, X. Peng, L. Z. Cai, A. M. Li, J. P. Guo, and Y. R. Wang, "Wavefront reconstruction and three-dimensional shape measurement by two-step dc-term-suppressed phase-shifted intensities," Opt. Lett. 34, 1210-1212 (2009).

15. N. T. Shaked, Y. Zhu, M. T. Rinehart, and A. Wax, "Twostep-only phase-shifting interferometry with optimized detector bandwidth for microscopy of live cells," Opt. Express 17, 15585-15591 (2009).

16. J.-P. Liu and T.-C. Poon, "Two-step-only quadrature phase-shifting digital holography," Opt. Lett. 34, 250-252 (2009).

17. J. Hahn, H. Kim, S.-W. Cho, and B. Lee, "Phase-shifting interferometry with genetic algorithm-based twin image noise elimination,” Appl. Opt. 47, 4068-4076 (2008). 